Research Article

Open Access

Special Issue Dedicated to Charles R. Johnson

Kenji Toyonaga*

\title{
The location of classified edges due to the change in the geometric multiplicity of an eigenvalue in a tree
}

https://doi.org/10.1515/spma-2019-0019

Received September 30, 2019; accepted November 29, 2019

\begin{abstract}
Given a combinatorially symmetric matrix $A$ whose graph is a tree $T$ and its eigenvalues, edges in $T$ can be classified in four categories, based upon the change in geometric multiplicity of a particular eigenvalue, when the edge is removed. We investigate a necessary and sufficient condition for each classification of edges. We have similar results as the case for real symmetric matrices whose graph is a tree. We show that a g-2-Parter edge, a g-Parter edge and a g-downer edge are located separately from each other in a tree, and there is a g-neutral edge between them. Furthermore, we show that the distance between a g-downer edge and a g-2-Parter edge or a g-Parter edge is at least 2 in a tree. Lastly we give a combinatorially symmetric matrix whose graph contains all types of edges.
\end{abstract}

Keywords: Edges, Eigenvalues, Graph, Multiplicity, Tree

MSC: 15A18, 05C50, 13H15, 05C05

\section{Introduction}

If $T$ is a simple, undirected tree on $n$ vertices, we denote the set of all $n$-by- $n$ real symmetric matrices by $\mathcal{S}(T)$, and the set of all combinatorially symmetric matrices (i.e. $A=\left(a_{i j}\right)$ and $a_{j i} \neq 0$ iff $a_{i j} \neq 0$ ) over a field $\mathbb{F}$ by $\mathcal{F}(T)$, the graph of whose off-diagonal entries is $T . \mathrm{m}_{A}(\lambda)$ and $\operatorname{gm}_{A}(\lambda)$ denote the algebraic multiplicity and geometric multiplicity of an eigenvalue $\lambda$ of $A$ respectively, and the set of eigenvalues of $A$ by $\sigma(A)$. When we remove a vertex $u$ from $T$, the remaining graph is denoted by $T(u)$, and corresponding submatrix of $A$ by $A(u)$, which is the principal submatrix of $A$, resulting from deletion of the row and column corresponding to $u$. When an edge $\{i, j\}$ is removed from $T$, we denote the remaining graph by $T\left(e_{i j}\right)$, and a corresponding matrix by $A\left(e_{i j}\right)$. When $T_{0}$ is a induced subgraph of $T, A\left[T_{0}\right]$ denotes the principal submatrix of $A$ corresponding to $T_{0}$. For an identified matrix $A \in \mathcal{F}(T)$, we often speak interchangeably about the graph and the matrix, for convenience.

Geometric multiplicity of an eigenvalue of $A \in \mathcal{F}(T)$ can change at most by 1 when a vertex is removed from $T$ [5, Lemma 1]. If $\operatorname{gm}_{A(v)}(\lambda)-\operatorname{gm}_{A}(\lambda)=1$ (resp. $0,-1$ ), then $v$ is called a geometrically Parter (resp. geometrically neutral, geometrically downer) vertex of $T$ for $\lambda$ in $A$, or g-Parter (resp. g-neutral, g-downer) for short. If $A$ is a Hermitian matrix, then we simply call Parter (resp. neutral, downer). We call the classification of a vertex as g-Parter, g-neutral or g-downer, the $g$-status of that vertex for a given eigenvalue of a matrix in $\mathcal{F}(T)$.

^Corresponding Author: Kenji Toyonaga: Department of Creative Engineering, National Institute of Technology, Kitakyushu College, Kokuraminami-ku, Kitakyushu, 802-0985, Japan: E-mail: toyonaga@kct.ac.jp 
Given $A \in \mathcal{F}(T)$, and $\lambda$ an eigenvalue of $A$, we may denote the g-status of a vertex in a natural numerical way, g-Parter should be 1 , g-neutral 0 and g-downer -1 . Specifically, define

$$
S_{A}(i)=\operatorname{gm}_{A(i)}(\lambda)-\operatorname{gm}_{A}(\lambda),
$$

so that the g-status number of a vertex is 1,0 and -1 , depending upon whether the vertex is g-Parter, g-neutral or g-downer.

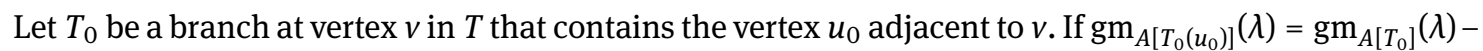
1 , then $T_{0}$ is called a $g$-downer branch at $v$ for the eigenvalue $\lambda$ and $u_{0}$ is called a g-downer neighbor at $v$ for $\lambda$ [6]. To identify a g-Parter vertex for $\lambda$ in $T$, a g-downer branch plays an important role in a tree.

Lemma 1. [6] Let $T$ be a tree, $v$ a vertex of $T, A \in \mathcal{F}(T)$ and $\lambda \in \sigma(A)$. Then $v$ is a g-Parter vertex for $\lambda$ if and only if there is a g-downer branch for $\lambda$ at $v$.

Next we consider the change in geometric multiplicity of an eigenvalue of $A \in \mathcal{F}(T)$ when an edge is removed from the tree $T$. Since removal of an edge from a graph may change the rank of a matrix by as much as 2 (in either direction), it may change the geometric multiplicity of an eigenvalue by as much as 2 (in either direction). So, we say that an edge $\{i, j\}$ is g-2-Parter (resp. g-Parter, g-neutral, g-downer, g-2-downer) for $\lambda$, if for $A \in \mathcal{F}(T)$ and $A\left(e_{i j}\right) \in \mathcal{F}\left(T\left(e_{i j}\right)\right)$,

$$
\operatorname{gm}_{A\left(e_{i j}\right)}(\lambda)-\operatorname{gm}_{A}(\lambda)=2(\text { resp. } 1,0,-1,-2) .
$$

In an analogous way, we may numerically classify edges:

$$
S_{A}\left(e_{i j}\right)=\operatorname{gm}_{A\left(e_{i j}\right)}(\lambda)-\operatorname{gm}_{A}(\lambda),
$$

so that the g-status number of an edge is $2,1,0,-1$ or -2 , depending upon whether the edge is g-2-Parter, $\mathrm{g}$-Parter, g-neutral, g-downer or g-2-downer. We note that there is a relation between the $\mathrm{g}$-status number of an edge and the g-status number of the incident vertices [8]:

$$
S_{A}\left(e_{i j}\right)=S_{A}(i)-S_{A\left(e_{i j}\right)}(i)=S_{A}(j)-S_{A\left(e_{i j}\right)}(j) .
$$

When $A \in \mathcal{F}(T)$ is Hermitian, the range of change in the algebraic multiplicity of an eigenvalue is investigated in [4], when one edge is removed from a tree $T$. Even when $A \in \mathcal{F}(T)$ is not Hermitian, we can observe that the range of change in the geometric multiplicity of an eigenvalue is the same as in Hermitian case, when one edge is removed from $T$. So we note that a g-2-downer edge does not occur in $A \in \mathcal{F}(T)$ when the graph is a tree. Because if $\operatorname{gm}_{A\left(e_{i j}\right)}(\lambda)=\operatorname{gm}_{A}(\lambda)-2$, then $i$ must be g-downer in $A$ and g-Parter in $A\left(e_{i j}\right)$ by (1), since the g-status number of g-2-downer edge is -2. Then, there must be a g-downer branch at $i$ in $A\left(e_{i j}\right)$ by Lemma 1. But, since $i$ is g-downer in $A$, there is no g-downer branch at $i$ in $A$, which is a contradiction. So, $\operatorname{gm}_{A\left(e_{i j}\right)}(\lambda)=\operatorname{gm}_{A}(\lambda)-2$ does not occur in $A \in \mathcal{F}(T)$.

Lemma 2. Let $T$ be a tree, $A \in \mathcal{F}(T), \lambda \in \mathbb{R}$ and $\{i, j\}$ an edge of $T$.

$$
\operatorname{gm}_{A}(\lambda)-1 \leq \operatorname{gm}_{A\left(e_{i j}\right)}(\lambda) \leq \operatorname{gm}_{A}(\lambda)+2 .
$$

Therefore an edge in a tree relative to $A \in \mathcal{F}(T)$ and an identified eigenvalue of $A$ can be classified in four classes based upon the change in the geometric multiplicity of a particular eigenvalue, when the edge is removed from $T$. For a real symmetric matrix $A \in \mathcal{S}(T)$ whose graph is a tree, the classification of edges in a tree was investigated regarding to the change in the algebraic multiplicity of a particular eigenvalue in [4] and [7] etc. Here, we consider combinatorially symmetric matrices whose graph is a tree, and we investigate the change in the geometric multiplicity of a particular eigenvalue when an edge is removed.

We give a necessary and sufficient condition for each classification of edges in a tree $T$ relative to $A \in \mathcal{F}(T)$. We have the similar results even in $\mathcal{F}(T)$ as the ones for the edges relative to $A \in \mathcal{S}(T)$. Furthermore, we clarified that a g-2-Parter edge, a g-Parter edge and a g-downer edge are not incident each other and that there 
is a g-neutral edge between them. We observe that the distance between a g-downer edge and a g-2-Parter edge or g-Parter edge is at least 2, that is, there are at least two g-neutral edges between them. We notice that these are also the similar results as the ones in Hermitian case.

Let $T$ be a tree, and $A \in \mathcal{F}(T)$. Let $v$ be a g-Parter vertex for an eigenvalue $\lambda$ of $A \in \mathcal{F}(T)$. If there is only one g-downer branch at $v$ for $\lambda$, we call $v$ a singly g-Parter vertex for $\lambda$, and if there is more than one g-downer branch at $v$, we call $v$ a multiply $g$-Parter vertex for $\lambda$.

When $A$ is a real symmetric matrix whose graph is a tree and $\lambda$ is an eigenvalue of $A$, a necessary and sufficient condition for each classified edge in $T$ relative to $A \in \mathcal{S}(T)$ and $\lambda$ is given in [4] or [7].

Theorem 3. [7] Let $T$ be a tree, $A \in \mathcal{S}(T), \lambda \in \mathbb{R}$ and $\{i, j\}$ an edge of $T$.

(i) The edge $\{i, j\}$ is 2-Parter for $\lambda$ if and only if $i$ and $j$ are both singly Parter for $\lambda$ in $A$, and each is the downer neighbor for the other.

(ii) The edge $\{i, j\}$ is Parter for $\lambda$ if and only if $i$ is singly Parter for $\lambda$ such that $j$ is the downer neighbor for $i$, and $j$ is neutral for $\lambda$, or vice versa for $i$ and $j$.

(iii) The edge $\{i, j\}$ is neutral for $\lambda \in \sigma(A)$ if and only if $i$ is Parter for $\lambda$ such that there is a downer branch at $i$ that does not contain $j$, or both $i$ and $j$ are neutral for $\lambda$ in $A$. Here $i$ and $j$ are interchangeable.

(iv) The edge $\{i, j\}$ is downer for $\lambda$ if and only if $i$ and $j$ are both downer vertices for $\lambda$ in $A$.

\section{Classification of edges by change in geometric multiplicity}

When $A$ is a combinatorially symmetric matrix whose graph is a tree $T$ and $\lambda$ is an eigenvalue of $A$, the geometric multiplicity of $\lambda$ of $A \in \mathcal{F}(T)$ can change when an edge in $T$ is removed. We consider a necessary and sufficient condition for each classified edge in $T$ relative to $A \in \mathcal{F}(T)$ and an identified eigenvalue of $A$. Here we note that we consider generally non-symmetric matrices $A \in \mathcal{F}(T)$.

Theorem 4. Let $T$ be a tree, $A \in \mathcal{F}(T), \lambda \in \mathbb{F}$ and $\{i, j\}$ an edge of $T$. The edge $\{i, j\}$ is $g$-2-Parter for $\lambda$ relative to $A$ if and only if $i$ and $j$ are both $g$-Parter for $\lambda$ in $A$, and each is the $g$-downer neighbor for the other.

Proof. For sufficiency, we suppose that $i$ and $j$ are g-Parter vertices relative to $A \in \mathcal{F}(T)$ for $\lambda$ and each is the g-downer neighbor for the other. Since $i$ and $j$ are both g-Parter in $A$ and they are both g-downer in $A\left(e_{i j}\right)$, the g-status number of the edge $e_{i j}$ becomes 2 by (1). For necessity, we suppose that $\{i, j\}$ is $\mathrm{g}$-2-Parter relative to $A$ for $\lambda$. Then, the status of $i$ and $j$ must be g-Parter in $A$ and g-downer in $A\left(e_{i j}\right)$ by (1). Since the graph is a tree, $j$ (resp. $i$ ) is g-downer in $A(i)$ (resp. $A(j)$ ).

Next, we consider conditions for a g-Parter edge and g-downer edge respectively for an eigenvalue relative to $A \in \mathcal{F}(T)$.

Theorem 5. Let $T$ be a tree, $A \in \mathcal{F}(T), \lambda \in \mathbb{F}$ and $\{i, j\}$ an edge of $T$. The edge $\{i, j\}$ is g-Parter for $\lambda$ relative to $A$ if and only if $i$ is $g$-Parter and $j$ is g-neutral for $\lambda$ in $A$, and $j$ is the $g$-downer neighbor for $i$. Here $i$ and $j$ are interchangeable.

Proof. For sufficiency, we suppose that $i$ is g-Parter and $j$ is g-neutral for $\lambda$ in $A$, and $j$ is the g-downer neighbor at $i$. Then, $j$ is g-downer in $A\left(e_{i j}\right)$ since $T$ is a tree. Thus the g-status number of the edge $\{i, j\}$ is 1 by (1). So the edge $\{i, j\}$ is g-Parter in $A$.

For the necessity portion, we suppose that the edge $\{i, j\}$ is g-Parter for $\lambda$ in $A$. Then, we can consider two possibilities of the g-status of the incident vertex, as g-neutral or g-Parter from (1). If the g-status of $i$ is $\mathrm{g}$-Parter, then the g-status of $i$ in $A\left(e_{i j}\right)$ is g-neutral. Thus, there is no g-downer branch for $\lambda$ at $i$ in $A\left(e_{i j}\right)$. Since $i$ was g-Parter in $A$, the g-downer branch at $i$ in $A$ has to include $j$. So $j$ is the g-downer neighbor for $i$. Since $T$ is a tree, $j$ is also g-downer in $A\left(e_{i j}\right)$. From (1), the g-status of $j$ in $A$ has to be g-neutral, since the edge $\{i, j\}$ is g-Parter. 
Next when the edge $\{i, j\}$ is g-Parter, if the g-status of $i$ is g-neutral, then the g-status of $i$ in $A\left(e_{i j}\right)$ is $g$ downer by (1). Since $T$ is a tree, $i$ is also g-downer in $A(j)$. Thus, $i$ is a g-downer neighbor for $j$ in $A$. Therefore, $j$ has to be $\mathrm{g}$-Parter by Lemma 1 . That concludes the assertion.

Theorem 6. Let $T$ be a tree, $A \in \mathcal{F}(T), \lambda \in \sigma(A)$ and $\{i, j\}$ an edge of $T$. The edge $\{i, j\}$ is $g$-downer for $\lambda$ relative to $A$ if and only if both $i$ and $j$ are $g$-downer vertices for $\lambda$ in $A$.

Proof. For sufficiency, we suppose that $i$ and $j$ are g-downer for $\lambda$ in $A$. To reach a contradiction, suppose that the edge $\{i, j\}$ is g-neutral or g-Parter for $\lambda$ in $A$. If the edge $\{i, j\}$ is g-neutral, then the g-status of $j$ in $A\left(e_{i j}\right)$ will be g-downer by (1). Then, $j$ is a g-downer neighbor for $i$ in $A$. Thus, $i$ must be $g$-Parter in $A$ by Lemma 1, a contradiction. Next, if the edge $\{i, j\}$ is g-Parter, then it is easy to find that the g-status of $i$ cannot be g-downer in $A$ from (1). So, the g-status of the edge is g-downer.

For necessity portion, we suppose that the edge $\{i, j\}$ is g-downer for $\lambda$ in $A$. From (1), the g-status of the incident vertex cannot be g-Parter. If $i$ is g-neutral in $A$, then the g-status of $i$ in $A\left(e_{i j}\right)$ has to be g-Parter by (1). Then there must be a g-downer branch at $i$ in $A\left(e_{i j}\right)$. But it is also a downer branch at $i$ in $A$, so $i$ must be g-Parter in $A$, a contradiction. Therefore, the incident vertices cannot be g-neutral in $A$. Thus, the g-statuses of the incident vertices have to be g-downer in $A$.

Next, we give a condition for a g-neutral edge for an eigenvalue relative to $A \in \mathcal{F}(T)$.

Theorem 7. Let $T$ be a tree, $A \in \mathcal{F}(T), \lambda \in \sigma(A)$ and $\{i, j\}$ an edge of $T$. The edge $\{i, j\}$ is g-neutral for $\lambda$ relative to $A$ if and only if there is a g-downer branch at $i$ that does not include $j$, or both $i$ and $j$ are g-neutral for $\lambda$ in $A$. Here $i$ and $j$ are interchangeable.

Proof. For sufficiency, we suppose that there is a g-downer branch at $i$ that does not include $j$. Then $i$ is $g$ Parter for $\lambda$ in $A$ from Lemma 1. We note that the branch is also a downer branch at $i$ in $A\left(e_{i j}\right)$. So, $i$ is g-Parter even in $A\left(e_{i j}\right)$. Therefore, the edge $\{i, j\}$ is g-neutral by (1). Next we suppose that both $i$ and $j$ are g-neutral for $\lambda$ in $A$. In this case, the edge $\{i, j\}$ cannot be g-2-Parter, g-Parter or g-downer from Theorem 4, 5 and 6 . So the edge $\{i, j\}$ is g-neutral for $\lambda$ in $A$.

For necessity portion, we suppose that the edge $\{i, j\}$ is g-neutral for $\lambda$ in $A$. In case that $i$ is g-Parter in $A, i$ is g-Parter in $A\left(e_{i j}\right)$ by (1), since the edge $\{i, j\}$ is g-neutral. Then there is a g-downer branch at $i$ that does not include $j$. In case that $i$ is g-downer in $A, i$ is g-downer in $A\left(e_{i j}\right)$. Then $i$ is a g-downer neighbor for $j$, so $j$ has to be g-Parter in $A$. We note that $j$ is also g-Parter in $A\left(e_{i j}\right)$, since the edge $\{i, j\}$ is g-neutral. Then there is a g-downer branch at $j$ that does not include $i$. Lastly, in case that $i$ is g-neutral in $A$, there is not possibility such that $j$ is g-downer in $A$, because if so, $j$ will be g-downer in $A\left(e_{i j}\right)$, then $i$ must be $g$-Parter, a contradiction. So, when $i$ is g-neutral, then $j$ is g-neutral or g-Parter. When $j$ is g-Parter in $A, j$ is g-Parter in $A\left(e_{i j}\right)$, then there is a g-downer branch at $j$ that does not include $j$. That conludes the assertion.

\section{Location of classified edges}

We consider the position of the classified edges in a tree. We observe some constraints about the positions of four kinds of edges in a tree.

Lemma 8. Let $T$ be a tree, $A \in \mathcal{F}(T)$ and $\lambda \in \mathbb{R}$. All edges adjacent to a g-2-Parter edge are g-neutral for $\lambda$ in $A$.

Proof. Let an edge $\{i, j\}$ be a g-2-Parter edge in $A$, and $\{i, k\}$ be another edge incident to $i$ in $A$. Then $i$ is gParter in $A$ and $j$ is a downer neighbor at $i$, from Theorem 4. Thus there is a g-downer branch at $i$ that does 
not include $k$. Then, the edge $\{i, k\}$ is g-neutral by Theorem 7. Here $i$ and $j$ are interchangeable, therefore all edges incident to a g-2-Parter edge are g-neutral in $A$.

We suppose that there is a g-Parter edge $\{i, j\}$ and a g-downer edge $\{k, l\}$ in $T$ relative to $A \in \mathcal{F}(T)$. Then, $i$ is g-Parter and $j$ is g-neutral in $T$ from Theorem 5, here $i$ and $j$ may be interchangeable. Since $k$ and $l$ are g-downers in $T$, from Theorem 6, a g-Parter edge and a g-downer edge cannot be incident in $T$.

Lemma 9. Let $T$ be a tree, $A \in \mathcal{F}(T)$ and $\lambda \in \sigma(A)$. A g-Parter edge and a g-downer edge are not incident each other in $T$.

From the two lemmas above, we have the information about the location of g-2-Parter edges, g-Parter edges and g-downer edges in $T$ for an eigenvalue relative to $A \in \mathcal{F}(T)$.

Theorem 10. Let $T$ be a tree, $A \in \mathcal{F}(T)$ and $\lambda \in \sigma(A)$. A g-2-Parter edge, a g-Parter edge and a g-downer edge for $\lambda$ in $T$ are located separately from each other, and there is at least one g-neutral edge between them.

Furthermore, we can investigate the distance between a g-downer edge and a g-2-Parter edge or g-Parter edge. We can observe that there are at least two g-neutral edges between them.

Theorem 11. Let $T$ be a tree, $A \in \mathcal{F}(T)$ and $\lambda \in \sigma(A)$. The distance between a g-downer edge and a g-2-Parter or a g-Parter edge is at least 2.

Proof. Let $\{i, j\}$ be a g-downer edge for $\lambda$ in $T$ relative to $A \in \mathcal{F}(T)$. Then, by Theorem $6, i$ and $j$ are g-downer vertices in $A$. A g-downer edge can be adjacent a g-neutral edge besides a g-downer edge. Suppose that $\{i, j\}$ is adjacent to a g-neutral edge $\{j, k\}$. Then, $k$ must be $\mathrm{g}$-Parter from Theorem 7 and $k$ is a g-multiply Parter vertex. Because $k$ is g-Parter in $A\left(e_{j k}\right)$, since $\{j, k\}$ is g-neutral in $A$ and the g-status of $k$ does not change after removing the edge $\{j, k\}$. So, there is a g-downer branch at $k$ that does not include $j$. On the other hand, $j$ is also g-downer in $A\left(e_{j k}\right)$ from the expression (1), since $\{j, k\}$ is g-neutral in $A$. Thus, there is a g-downer branch at $k$ that includes $j$. Then $k$ is a multiply g-Parter vertex in $A$.

Next we observe that all edges incident to $k$ are g-neutral. Let $\{k, l\}$ be an edge incident to $k$. Then, there is a downer branch at $k$ that does not include $l$, since $k$ is a multiply g-Parter vertex in $A$. Thus, $\{k, l\}$ is a $g$ neutral edge by Theorem 7 . So, two g-neutral edges $\{j, k\}$ and $\{k, l\}$ can be incident to a g-downer edge $\{i, j\}$. That means that there are at least two neutral edges between a g-neutral edge and a g-2-Parter or g-Parter edge in $T$ relative to $A$.

From the above Theorem, we can deduce the next corollary.

Corollary 12. Let $T$ be a tree, $A \in \mathcal{F}(T)$ and $\lambda \in \mathbb{R}$. If the diameter of $T$ is less than 4, then a g-downer edge and a g-2-Parter or a g-Parter edge are not contained together in $T$.

Next we give an $A \in \mathcal{F}(T)$ whose graph is a tree that contains all kind of edges.

Example 13. Let

$$
A=\left[\begin{array}{llllllll}
2 & 1 & 0 & 0 & 0 & 0 & 0 & 0 \\
1 & 2 & 1 & 0 & 0 & 0 & 0 & 0 \\
0 & 1 & 5 & 1 & 1 & 0 & 0 & 0 \\
0 & 0 & 1 & 3 & 0 & 0 & 0 & 0 \\
0 & 0 & 1 & 0 & 3 & 1 & 0 & 0 \\
0 & 0 & 0 & 0 & 1 & 3 & 1 & 0 \\
0 & 0 & 0 & 0 & 0 & 1 & 4 & 1 \\
0 & 0 & 0 & 0 & 0 & 0 & 1 & 3
\end{array}\right]
$$

whose graph is as follows. 


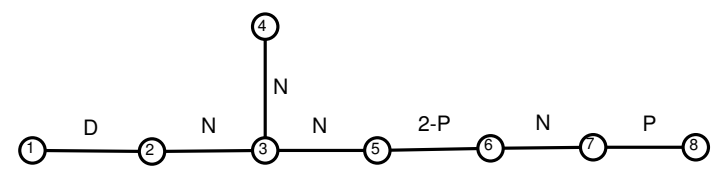

$A$ has the eigenvalue 3 with multiplicity 1 . When one edge is removed from the tree, we can observe the change in multiplciity of the eigenvalue 3 so that $\operatorname{gm}_{A\left(e_{12}\right)}(3)=0, \operatorname{gm}_{A\left(e_{23}\right)}(3)=\operatorname{gm}_{A\left(e_{34}\right)}(3)=\operatorname{gm}_{A\left(e_{35}\right)}(3)=$ $\operatorname{gm}_{A\left(e_{67}\right)}(3)=1, \operatorname{gm}_{A\left(e_{56}\right)}(3)=3$ and $\operatorname{gm}_{A\left(e_{78}\right)}(3)=2$. The character over each edge denotes the $\mathrm{g}$-status of the edge (2-P: g-2-Parter, P: g-Parter, N: g-neutral and D: g-downer). The matrix $A$ has all kind of edges in the tree, and we can observe that there is at least one g-neutral edge between a g-2-Parter, g-Parter and g-downer edge, further we can see that there are two g-neutral edges beween a g-downer edge and a g-2-Parter edge in a tree.

Acknowledgements: The author thanks to the anonymous referee for the careful reading and comments that helped to improve the original version of the manuscript.

\section{References}

[1] R. Horn, C.R.Johnson, Matrix Analysis, $2^{\text {nd }}$ Edition. Cambridge University Press, New York, 2013.

[2] C.R. Johnson, A. Leal-Duarte and C.M. Saiago, The Parter-Wiener theorem: refinement and generalization, SIAM J. Matrix Anal. Appl. 25 (2003), 352-361.

[3] C.R. Johnson, A. Leal-Duarte, C.M. Saiago. The change in eigenvalue multiplicity associated with perturbation of a diagonal entry, Linear Multilinear Algebra 60 (2012), 525-532.

[4] C.R. Johnson, P.R. McMichael, The change in multiplicity of an eigenvalue of a Hermitian matrix associated with the removal of an edge from its graph, Discrete Math. 311 (2011), 166-170.

[5] C.R. Johnson, C. M. Saiago, Geometric Parter-Wiener, etc. theory, Linear Algebra Appl, 537 (2018), 332-347.

[6] C.R. Johnson, C.M. Saiago, Eigenvalues, Multiplicities and Graphs. Cambridge University Press, New York, 2018.

[7] K. Toyonaga, C.R. Johnson, The classification of edges and the change in multiplicity of an eigenvalue of a real symmetric matrix tesulting from the change in an edge value, Spec. Matrices 5 (2017), 51-60.

[8] C.R. Johnson, C.M. Saiago, K. Toyonaga, Classification of vertices and edges with respect to the geometric multiplicity of an eigenvalue in a matrix, with a given graph, over a field, Linear Multilinear Algebra 66 (2018), 2168-2182. 